\title{
Post-Molar Stage I Low-Risk Gestational Trophoblastic Neoplasia: Transvaginal Ultrasound Findings and Their Correlation with Chemotherapy Response
}

\author{
Behnaz Moradi ${ }^{1}$, Ali Borhani ${ }^{2,}{ }^{*}$, Fariba Yarandi ${ }^{3}$, Maryam Rahmani ${ }^{2}$, Elham Shirali ${ }^{3}$, Mahrooz \\ Malek $^{2}$, Nasim Batavani ${ }^{2}$ and Mohamad Ali Kazemi ${ }^{4}$ \\ ${ }^{1}$ Department of Radiology, Women' Yas Hospital, Tehran University of Medical Sciences, Tehran, Iran \\ ${ }^{2}$ Advanced Diagnostic and Interventional Radiology Research Center (ADIR), Imam Khomeini Hospital, Tehran University of Medical Sciences, Tehran, Iran \\ ${ }^{3}$ Department of Gynecologic Oncology, Women' Yas Hospital, Tehran University of Medical Sciences, Tehran, Iran \\ ${ }^{4}$ Department of Radiology, Amiralam Hospital, Tehran University of Medical Sciences, Tehran, Iran \\ "Corresponding author: Department of Radiology, Imam Khomeini Hospital, Tehran University of Medical Sciences, Keshavars Boulevard, Tehran, Iran. Tel: +98-9155016311, \\ Email: aliborhani@gmail.com
}

Received 2019 April 06; Revised 2019 November 26; Accepted 2019 November 30.

\begin{abstract}
Background: Early diagnosis of gestational trophoblastic neoplasia and its complications are pivotal for prompt and efficacious treatment. Transvaginal pelvic ultrasound could detect myometrial invasion and endometrial thickening following dilation and evacuation of hydatiform mole and also in the assessment of response to chemotherapy.

objectives: In this study we aimed to investigate transvaginal ultrasound findings of stage I low-risk gestational trophoblastic neoplasia (GTN) and whether there is an association between ultrasound findings and chemotherapy response.

Patients and Methods: This study included 31 consecutive patients with postmolar stage I low-risk GTN. We recorded International Federation of Gynecology and Obstetrics (FIGO) score, and transvaginal ultrasound findings including color and pulsed Doppler interrogation at the time of beta human chorionic gonadotropin ( $\beta$-hCG) rise. The number of Act-D cycles that each patient needed to achieve complete remission was also recorded.

Results: Of the 31 patients with post-evacuation trans-vaginal ultrasound evaluation, 2 (6.5\%) patients had no detectable finding, 4 $(12.9 \%)$ had lesions limited to the endometrium, 12 (38.7\%) had lesions with < 50\% invasion into myometrium, 7 (22.6\%) had lesions with $>50 \%$ invasion into myometrium, $4(12.9 \%)$ had lesions that reached uterine serosal surface and $2(6.5 \%)$ had arteriovenous malformation (AVM)-like myometrial lesions. The number of Act-D cycles patients needed to achieve remission was 6 cycles in patients with no finding, lesion limited to endometrium and less than $50 \%$ myometrial invasion and was 8 cycles in patients with $>$ $50 \%$ invasion \pm involvement of serosal surface. One patient in first group and two in second group need multi-agent chemotherapy. But these differences were not significant $(\mathrm{P}=0.172)$.

Conclusion: There was a non-significant increase in treatment duration and need of multiagent chemotherapy with more extensive ultrasound findings among patients with stage I low risk GTN.
\end{abstract}

Keywords: Gestational Trophoblastic Neoplasia, Actinomycin D, Transvaginal Ultrasound

\section{Background}

Post-molar gestational trophoblastic neoplasia (GTN) is commonly diagnosed in asymptomatic patients with risen beta human chorionic gonadotropin ( $\beta$-hCG) levels after suction-evacuation of a molar pregnancy. Early diagnosis of GTN and its complications are pivotal in prompt and efficacious treatment as well as conservation of fertility. Clinical findings, serial quantitative $\beta$-hCG titers and trans-vaginal ultrasound evaluation of pelvis are the mainstay of initial diagnosis of this condition (1). Pelvic ultra- sound and Doppler assessment are performed in patients with elevated $\beta$-hCG levels after dilation and suction evacuation for molar pregnancy to rule out pregnancy, assess uterine volume or possible extension of disease into the pelvic cavity (2).

Locally invasive gestational trophoblastic neoplasms are seen at gray-scale ultrasound as nonspecific focal masses located inside the myometrium with a variable endometrial component (3). Ultrasound might show a hypoechoic or echogenic complex or multicystic mass with 
anechoic spaces which results from hemorrhage, necrosis, cysts, or vascular spaces (4). An enlarged heterogeneous uterus with lobulated borders or a large non-specific pelvic mass with invasion of other pelvic organs is indicative of a more extensive GTN (5). High levels of circulating $\beta$-hCG induce multilocular cystic appearance of both ovaries as a result of formation of multiple bilateral large $(2-3 \mathrm{~cm})$ theca lutein cysts (6). Doppler ultrasound demonstrates low-resistance high-velocity arterial flow and arterio-venous malformation (AVM) within the myometrium that has been proved to have a role in confirming the diagnosis of GTN. Extension of low-resistive arterial flow from endometrium into the myometrium is suggestive of invasive mole (3). Uterine artery pulsatility index (PI) of less than 1.5 and resistive index (RI) of less than 0.5 are indicative of low uterine artery resistance and have been observed in GTN (3).

Owing to excellent chemosensitivity of GTN, the mainstay of management of this disease is with chemotherapy, and treatment of women with postmolar International Federation of Gynecology and Obstetrics (FIGO) stage I lowrisk GTN (a score of $\leq 6$ WHO risk factor scoring system) is by single-agent chemotherapy (7). Polychemotherapy of invasive mole is reserved for patients who develop singleagent drug chemoresistance, which is associated with significant side effects, risk of premature failure of ovaries and growth of secondary tumors (8). There are studies that suggest that transvaginal pelvic ultrasound can detect myometrial invasion and endometrial thickening following dilation and evacuation of hydatiform mole and it could also be employed in thee assessment of response to chemotherapy (9-11)

\section{Objectives}

In this study, we aimed to investigate transvaginal ultrasound findings of stage I low-risk GTN and whether there is an association between ultrasound findings and chemotherapy response.

\section{Patients and Methods}

This cross-sectional study that was performed between August 2017 and July 2018, included 31 consecutive patients with postmolar stage I low-risk GTN who were diagnosed based on rising or persistent levels of HCG following uterine evacuation of hydatidiform mole. The study was approved by the Institutional Committee of Research Ethics and obtaining informed consent from patients was unnecessary because transvaginal pelvic ultrasound and Doppler assessment is a part of the routine protocol in evaluation of patients with GTN. A power test was performed and a minimum sample size of 26 patients was found to be necessary to provide $80 \%$ statistical power. Patients who were diagnosed as high risk (score of 7 or more according to the FIGO 2000 scoring system) or metastatic disease in pre-treatment evaluations (four patients) were excluded from the study. Pre-treatment assessments comprised of abdomino-pelvic computed tomography (CT) scans, transvaginal pelvic ultrasound (TVS), and serial $\beta$-hCG measurements. All the patients had confirmed histologic diagnosis and were treated with single-agent chemotherapy. Data collected from patients' records included age, gestational age at the time of diagnosis of hydatidiform mole, parity history, time interval between uterine evacuation and rise of $\beta$-hCG, pre-evacuation HCG level, FIGO score, treatment protocol and sonographic findings including color and pulsed Doppler interrogation.

Ultrasound was performed using a 4-9-MHz endovaginal probe for trans-vaginal examination (ACUSON X300 ultrasound system, USA) and a 2-6-MHz curve probe for trans-abdominal examination. All patients first underwent trans-abdominal examination to obtain a general view of the pelvic cavity followed by trans-vaginal examination to enable a more detailed evaluation of the uterus and adnexa. Power Doppler was employed to detect presence of increased intra- and perilesional blood flow compared with the surrounding normal tissue. Identified lesions were classified into six types: no detectable lesion, lesions limited to the endometrium, lesions with $<50 \%$ invasion into the myometrium (Figure 1), lesions with $>$ $50 \%$ invasion into the myometrium but without uterine serosal surface involvement, lesions with invasion of uterine serosal surface (Figure 2) and lesions with $>50 \%$ invasion and intense hypervascularity (arteriovenous malformation [AVM] like). Then the first three types were included in group A and the second three types were included in group $\mathrm{B}$.

Pulsatility index (PI) of uterine arteries was measured using pulsed wave Doppler with a $2 \mathrm{~mm}$ sample volume and at an angle of insonation $<30^{\circ}$ in the ascending branch of the uterine artery at its paracervical portion. Three consecutive waveforms were obtained and the assessment was repeated on the opposite, the lower PI was recorded. Presence of ovarian theca lutein cysts $(>30 \mathrm{~mm})$ were also recorded. All the patients were treated by Actinomycin (act-D) $1.25 \mathrm{mg} / \mathrm{m}^{2}$ intravenous every 2 weeks. Treatment were maintained in all patients, until the hCG level has returned to normal and stayed normal for a further 6 weeks. The number of cycles that each patient needed to 


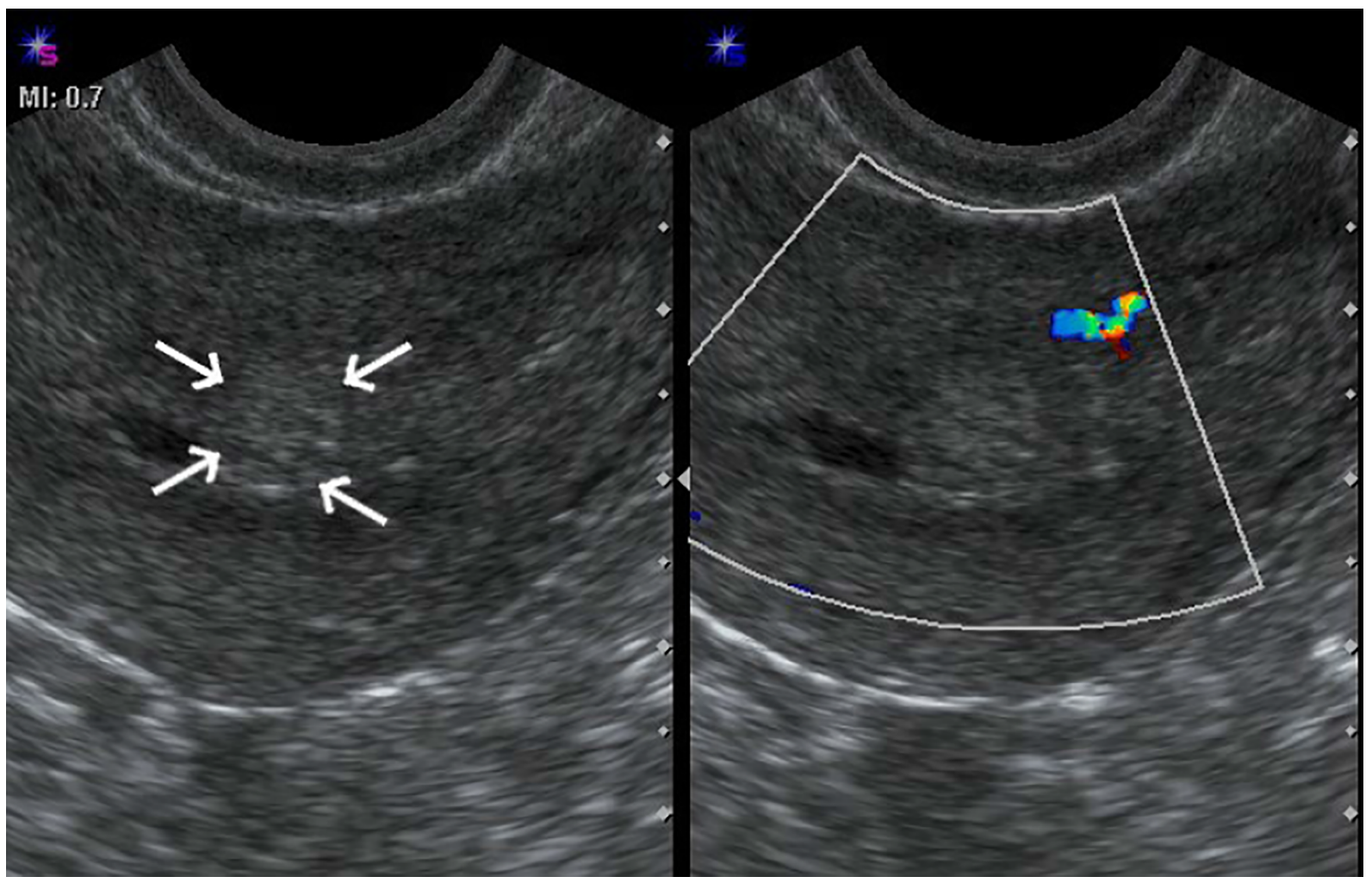

Figure 1. Grayscale (left) and color Doppler (right) transvaginal images of an invasive mole in a patient with gestational trophoblastic neoplasia show a hypovascular intramural lesion (arrows) with $<50 \%$ invasion into the myometrium.

achieve complete remission were recorded.

Association between various ultrasound findings and chemotherapy response were assessed by using IBM SPSS Statistics for Windows Version 22 (IBM Corp, released 2013, IBM statistics for Windows, version 22, Armonk, New York). Normality of distributions of various variables including patients' age, uterine artery resistance, FIGO score and $\beta$ hCG levels were assessed using Kolmogorov-Smirnov test. Analysis of data using Pearson's chi-squared and KruskalWallis tests were performed for analysis of association between the number of required chemotherapy sessions and ultrasound findings including tumor size, tumor hypervascularity, uterine artery resistive index, and FIGO score. Differences with $\mathrm{P}<0.05$ were statistically significant.

\section{Results}

The mean age of the patients was $28.6 \pm 6.5$ years (1852 years). Serum human chorionic gonadotropin (HCG) levels were raised in all patients at first referral, with levels measuring up to $1073430 \mathrm{ng} / \mathrm{mL}$ with an average of $280605.7 \mathrm{ng} / \mathrm{mL}$. The mean gestational age at the time of evacuation was 9 weeks (range, 6 - 22 weeks of gestation) with one patient (3.2\%) with a FIGO score of 1, five (16.1\%) scoring 2, five (16.1\%) scoring 3, 14 (45.2\%) scoring 4 and six (19.4\%) scoring 5. GTN was diagnosed following complete mole in $22(70 \%)$ patients, partial mole in seven (22.5\%) patients and two (6.4\%) patients had a normal pregnancy and twin pregnancy. The mean uterine artery PI was $1.53 \pm 0.62$ $(0.27-2.4)$. Of the 31 patients with post-evacuation transvaginal ultrasound evaluation, two (6.5\%) patients had no detectable finding, four (12.9\%) had lesions limited to the endometrium, $12(38.7 \%)$ had lesions with $<50 \%$ invasion into the myometrium, seven (22.6\%) had lesions with $>50 \%$ invasion into myometrium, four (12.9\%) had lesions that reached the uterine serosal surface and two (6.5\%) had > $50 \%$ invasion with AVM-like lesions. The first three types that were included in group A comprised of 18 (58\%) patients and the second three types that were included in group B comprised of 13 (42\%) patients. The difference of gestational age at the time of diagnosis between groups $A$ and $\mathrm{B}$ was statistically significant $(\mathrm{P}=0.008)$. In group $\mathrm{A}$, gestational age ( $9.75 \pm 1.75$ weeks) was higher than group B $(8.73 \pm 4.71$ weeks $)$. Clinical and ultrasound features are 


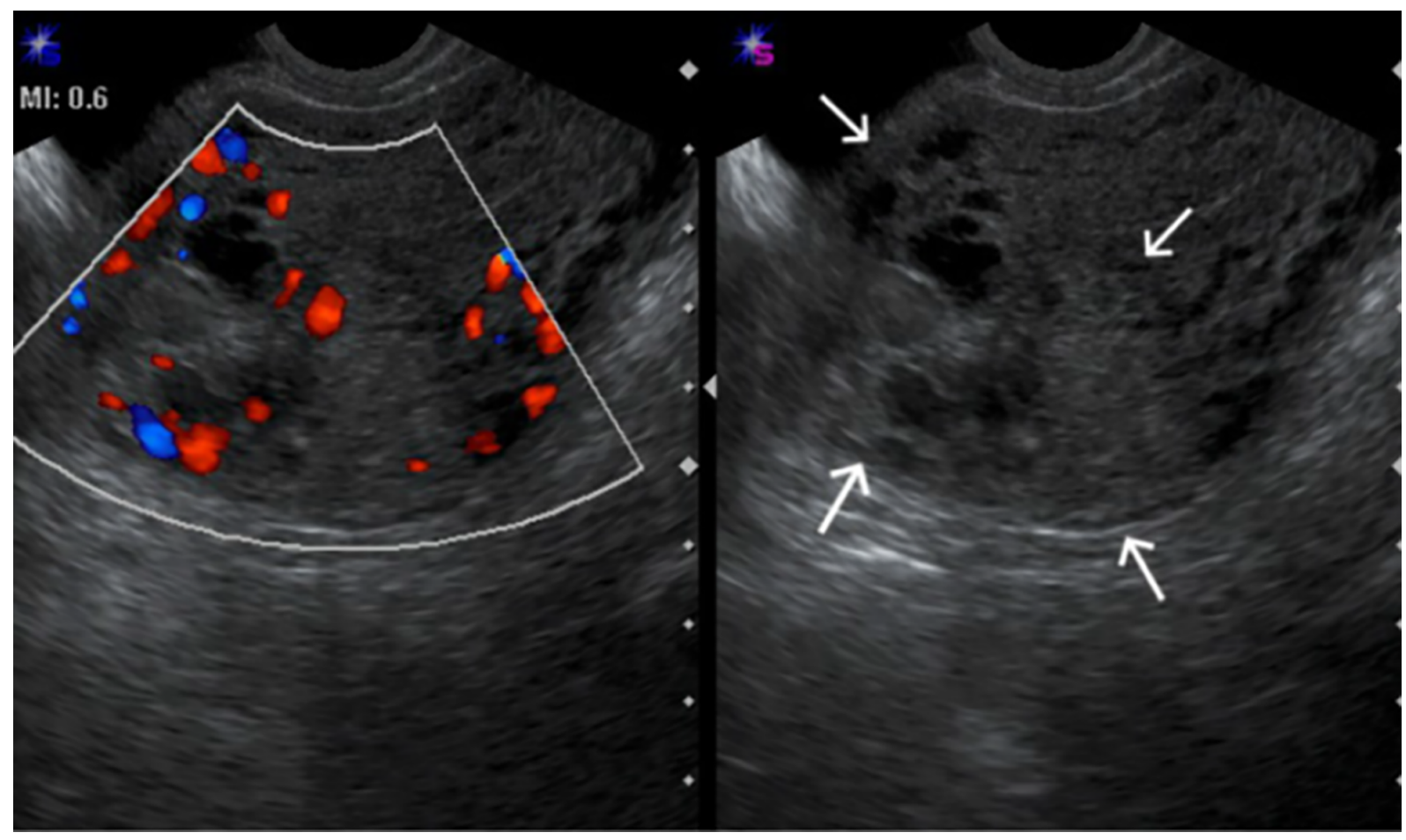

Figure 2. Grayscale (right) and color Doppler (left) transvaginal images of an invasive mole in a patient with gestational trophoblastic neoplasia show a bulky mass (arrows) with areas of vascularity that have invaded the entire uterine wall from the endometrium to the serosal surface of the uterus.

\begin{tabular}{lc}
\hline \begin{tabular}{l} 
Table 1. Clinical and Ultrasound Features of Patients with Post-Molar Stage $1 \mathrm{GTN}^{\mathrm{a}}$ \\
\hline Clinical feature
\end{tabular} & Value \\
\hline Age & 28 years $(18-52)$ \\
$\begin{array}{l}\text { Gestational age at the time of } \\
\text { diagnosis }\end{array}$ & 9 weeks $(6-22)$ \\
\hline $\begin{array}{l}\text { Uterine artery PI } \\
\text { HCG level at the time of diagnosis }\end{array}$ & $1.53(0.27-2.28)$ \\
\hline Lesion size & $280605 \mathrm{ng} / \mathrm{mL}(10000-1073430)$ \\
\hline Act-D cycles & $23.79 \pm 14.85 \mathrm{~mm}(6-68)$ \\
\hline
\end{tabular}

Abbreviations: HCG, human chorionic gonadotropin; GTN, gestational trophoblastic neoplasia; PI, pulsatility index

${ }^{\mathrm{a}}$ Data are presented as mean (range) or mean $\pm \mathrm{SD}$ (range).

summarized in Tables 1 and 2.

The difference of uterine artery PI between groups A and $\mathrm{B}$ was statistically different $(\mathrm{P}=0.002)$. Uterine artery PI in group A $(1.87 \pm 0.49)$ was significantly higher than group B (0.98 \pm 0.47$)$. There was no significant association between ultrasound findings and FIGO scoring ( $\mathrm{P}$ $=0.232$ ). The number of Act-D cycles patients needed to achieve remission was $6.83 \pm 2.68$ ( 3 - 14 cycles). A significant association was found between lesion size $(23.79 \mathrm{~mm}$ $\pm 14.85 \mathrm{~mm}$ ) in ultrasound and the number of chemotherapy cycles. Larger lesions were more likely to need more Act-D cycles to resolve $(\mathrm{P}=0.019)$. No significant association was found between lesion hypervascularity in ultrasound and chemotherapy response $(\mathrm{P}=0.434)$. There was a non-significant increase $(\mathrm{P}=0.085)$ in treatment duration in patients with more extensive ultrasound findings. The patients' treatment duration in group B was $8 \pm 3.41 \mathrm{cy}-$ cles more than group A with $6 \pm 1.28$ cycles. The duration of treatment was longest in patients with uterine serosal invasion (none cycles). One patient in group A and two in the second group needed multi-agent chemotherapy; however, these differences were not significant $(\mathrm{P}=0.172)$. There was no significant difference in the number of patients with FIGO score 5 between the two groups. Statistical analysis results are reported in Tables 3 and 4.

\section{Discussion}

Post-molar low risk GTN (FIGO prognostic score of $\leq 6)$ is considered as an uncommon disease, which in most cases is curable by uterine evacuation followed by single-agent chemotherapy. Aside from serum hCG levels, ultrasound as a widespread available imaging tool 
Table 2. FIGO Scoring and Categorized Ultrasound Features of Patients with PostMolar Stage 1 GTN

\begin{tabular}{|c|c|}
\hline & Number of patients \\
\hline \multicolumn{2}{|l|}{ FIGO scoring } \\
\hline 1 & 1 \\
\hline 2 & 5 \\
\hline 3 & 5 \\
\hline 4 & 14 \\
\hline 5 & 6 \\
\hline \multicolumn{2}{|l|}{ Ultrasound feature } \\
\hline Type 0 (no detectable lesion) & 2 \\
\hline Type 1 (lesions limited to the endometrium) & 4 \\
\hline $\begin{array}{l}\text { Type } 2 \text { (lesions with }<50 \% \text { invasion into the } \\
\text { myometrium) }\end{array}$ & 12 \\
\hline $\begin{array}{l}\text { Type } 3 \text { (lesions with }>50 \% \text { invasion into the } \\
\text { myometrium) }\end{array}$ & 7 \\
\hline $\begin{array}{l}\text { Type } 4 \text { (lesions with invasion of the uterine } \\
\text { serosal surface) }\end{array}$ & 4 \\
\hline $\begin{array}{l}\text { Type } 5 \text { (lesions with }>50 \% \text { invasion and } \\
\text { intense hypervascularity [AVM- like]) }\end{array}$ & 2 \\
\hline
\end{tabular}

Abbreviations: AVM, arteriovenous malformation; FIGO, International Federation of Gynecology and Obstetrics; GTN, gestational trophoblastic neoplasia; $\mathrm{SD}$, standard deviation

Table 3. Comparison of Ultrasound Features and FIGO Score with Chemotherapy Response in Patients with Post-Molar Stage $1 \mathrm{GTN}^{\mathrm{a}}$

\begin{tabular}{|c|c|c|}
\hline & Act-D cycles & Pvalue \\
\hline FIGO score & & 0.100 \\
\hline 1 & - & \\
\hline 2 & $5.33 \pm 0.58$ & \\
\hline 3 & $7.25 \pm 2.50$ & \\
\hline 4 & $7.91 \pm 3.18$ & \\
\hline 5 & $5.33 \pm 1.51$ & \\
\hline Ultrasound feature & & 0.085 \\
\hline Type 0 (no detectable lesion) & 0 & \\
\hline $\begin{array}{l}\text { Type } 1 \text { (lesions limited to the } \\
\text { endometrium) }\end{array}$ & $4.00 \pm 1.41$ & \\
\hline $\begin{array}{l}\text { Type } 2 \text { (lesions with }<50 \% \text { invasion into } \\
\text { the myometrium) }\end{array}$ & $6.40 \pm 0.84$ & \\
\hline $\begin{array}{l}\text { Type } 3 \text { (lesions with }>50 \% \text { invasion into } \\
\text { the myometrium) }\end{array}$ & $7.29 \pm 3.45$ & \\
\hline $\begin{array}{l}\text { Type } 4 \text { (lesions with invasion of the } \\
\text { uterine serosal surface) }\end{array}$ & $9.25 \pm 3.45$ & \\
\hline $\begin{array}{l}\text { Type } 5 \text { (lesions with }>50 \% \text { invasion and } \\
\text { intense hypervascularity [AVM- like]) }\end{array}$ & $4.00 \pm 0.00$ & \\
\hline
\end{tabular}

Abbreviations: FIGO, International Federation of Gynecology and Obstetrics; GTN, gestational trophoblastic neoplasia; SD, standard deviation

${ }^{\text {a }}$ Values are expressed as mean $\pm S D$
Table 4. Univariate Analysis of Different Clinical and Ultrasound Features Between Group A and Group B ${ }^{\mathrm{a}}$

\begin{tabular}{lccc}
\hline & Group A & Group B & P value \\
\hline Act-D cycles & $6.00 \pm 1.28$ & $8.00 \pm 3.41$ & 0.172 \\
\hline Gestational age (wk) & $9.75 \pm 1.75$ & $8.73 \pm 4.71$ & 0.008 \\
\hline Uterine artery PI & $1.87 \pm 0.49$ & $0.98 \pm 0.47$ & 0.002 \\
\hline Lesion size (mm) & $14.38 \pm 7.27$ & $35.18 \pm 14.4$ & 0.019 \\
\hline FIGO score 5 & 2 & 3 & 0.655 \\
\hline $\begin{array}{l}\text { Time of hCG rise after } \\
\text { evacuation }(\mathbf{w k})\end{array}$ & $5.44 \pm 2.15$ & $4.75 \pm 1.75$ & 0.391 \\
\hline $\begin{array}{l}\text { Pre-evacuation } \beta \text {-hCG } \\
\text { level (ng/mL) }\end{array}$ & $292304 \pm 300323$ & $323327 \pm 312651$ & 0.793 \\
\hline
\end{tabular}

Abbreviations: FIGO, International Federation of Gynecology and Obstetrics; HCG, human chorionic gonadotropin; PI, pulsatility index; SD, standard deviation; wk, week

${ }^{a}$ Values are expressed as mean $\pm \mathrm{SD}$.

has evolved into a standard method in confirming hydatidiform mole, evaluation of possible myometrial invasion and response to chemotherapy (3). Few studies have assessed correlations between ultrasound findings and single-agent chemotherapy effects in post-molar GTN, and even less data is available regarding the correlation of ultrasound finding and resistance to Act-D (12).

Invasive mole in transvaginal ultrasonography (TVS) is demonstrated by endometrial thickening or areas of hyper-echogenicity in the myometrium with or without associated hyper-vascularity in Doppler assessment (3). In this study, we categorized TVS findings into six different types and two groups and found a non-significant increase in treatment duration among patients with more extensive ultrasound findings, and also, multi-agent chemotherapy is more likely needed in this group. Our findings also showed that patients with larger lesions in ultrasound needed more Act-D cycles to achieve remission. These findings are in agreement with previous research in this field that suggests TVS is a good predictor of response to chemotherapy in GTN $(9,10)$. This is particularly important in identifying the group of patients who would routinely undergo second-line treatment with the indication of chemo-resistance, but in whom further single agent chemotherapy may lead to a complete response, avoiding the significant adverse effects of multi-agent chemotherapy (9). In a recent study conducted by Lei et al., which assessed the effects of Actinomycin D (Act-D) on post-molar GTN, authors reported that existing invasive uterine lesions and their size in ultrasound had the most effective prediction effects for resistance to Act-D (12). Ultrasound could be also employed in monitoring tumor response to Methotrexate (MTX), because it could readily show reduc- 
tion of tumor perfusion using Doppler analysis and recognize decreasing lesion echogenicity (9). This could be particularly helpful in patients with signs of chemo-response in ultrasound but with HCG plateau, that are routinely considered drug-resistant.

In GTN similar to normal pregnancies, invasion of decidua basalis by trophoblasts and progressive erosion of uterine spiral arteries lead to a decrease in utero-placental vascular resistance and increase in diastolic blood flow of uterine arteries (13). Doppler ultrasound provides a noninvasive technique for the quantitative assessment of these changes by velocimetry indices based on blood flow waveforms. PI has been found to be the Doppler parameter that is most strongly associated with the risk of post-molar GTN as well as MTX resistance (14). Our data showed that more extensive ultrasound findings are associated with a lower uterine artery PI which is justified by deeper myometrial neoplastic invasion and myometrial vascularization. Lower uterine artery resistance at diagnosis is also associated with a longer treatment duration. Agrawal et al. assessed 239 patients with low-risk GTN for both uterine artery PI and MTX resistance, and found uterine artery PI to be an independent factor in the prediction of MTX resistance in these patients (15). Authors concluded that upfront multi-agent chemotherapy could be considered for patients with low-risk GTN and a uterine artery PI $\leq 1$ (15). Comparably, in a prospective study of 40 patients with GTN by Long et al., uterine artery PI cut-off value of $\leq 1.1$ was proposed to be included in the prognostic scoring system of trophoblastic tumors (16). There are several other studies that support the application of Doppler ultrasound as a useful tool in the prediction of MTX resistance $(14,17,18)$. However, a possible correlation between uterine artery vascularization and resistance to Act-D is yet to be assessed. Lei et al. found existing invasive uterine lesions in prechemotherapy TVS, FIGO score $\geq 5$, and pre-chemotherapy hCG $\geq 4000 \mathrm{IU} / \mathrm{L}$ to be independent factors for resistance to Act-D, but uterine artery Doppler indices were not explored by the study (12).

The present study had a number of limitations. First, our small sample size restricts further extrapolation of its findings and thus larger cohort studies are necessary to confirm our results. Second, the retrospective design of this study is inherently associated with selection bias and recall bias.

In the future, ultrasound imaging could help patient management regarding the selection of treatment plan and more than that, TVS findings could be incorporated into FIGO scoring system, so that patients with certain TVS findings and large lesions might be upstaged and con- sequently offered poly-chemotherapy rather than single agent chemotherapy with MTX or Act-D. Further research and larger studies are required to establish imaging findings that are associated with chemo-resistance and possible implantation of ultrasound findings in FIGO scoring.

\section{Footnotes}

Authors' Contribution: Study concept and design: Behnaz Moradi and Ali Borhani; analysis and interpretation of data: Nasim Batavani and Fariba Yarandi; drafting of the manuscript: Ali Borhani; critical revision of the manuscript for important intellectual content: Maryam Rahmani, Elham Shirali, and Mahrooz Malek; statistical analysis: Mohamad Ali Kazemi

Conflict of Interests: The authors declare that there is no conflict of interest regarding publication of this article.

Ethical Approval: This study has been approved by the ethics committee of Tehran University of Medical Sciences (code number: 93-02-98-26039).

Funding/Support: This study did not receive any funding.

\section{References}

1. Lurain JR. Gestational trophoblastic disease II: Classification and management of gestational trophoblastic neoplasia. Am J Obstet Gynecol. 2011;204(1):11-8. doi: 10.1016/j.ajog.2010.06.072. [PubMed: 20739008].

2. Mangili G, Lorusso D, Brown J, Pfisterer J, Massuger L, Vaughan M, et al. Trophoblastic disease review for diagnosis and management: A joint report from the International Society for the Study of Trophoblastic Disease, European Organisation for the Treatment of Trophoblastic Disease, and the Gynecologic Cancer InterGroup. Int J Gynecol Cancer. 2014;24(9 Suppl 3):S109-16. doi: 10.1097/IGC.0000000000000294. [PubMed: 25341573].

3. Zhou Q, Lei XY, Xie Q, Cardoza JD. Sonographic and Doppler imaging in the diagnosis and treatment of gestational trophoblastic disease: A 12-year experience. J Ultrasound Med. 2005;24(1):15-24. doi: 10.7863/jum.2005.24.1.15. [PubMed: 15615924].

4. Dhanda S, Ramani S, Thakur M. Gestational trophoblastic disease: A multimodality imaging approach with impact on diagnosis and management. Radiol Res Pract. 2014;2014:842751. doi: 10.1155/2014/842751. [PubMed: 25126425]. [PubMed Central: PMC4122023].

5. Kani KK, Lee JH, Dighe M, Moshiri M, Kolokythas O, Dubinsky T. Gestatational trophoblastic disease: Multimodality imaging assessment with special emphasis on spectrum of abnormalities and value of imaging in staging and management of disease. Curr Probl Diagn Radiol. 2012;41(1):1-10. doi: 10.1067/j.cpradiol.2011.06.002. [PubMed: 22085657].

6. Brown DL, Dudiak KM, Laing FC. Adnexal masses: US characterization and reporting. Radiology. 2010;254(2):342-54. doi: 10.1148/radiol.09090552. [PubMed: 20089722]. 
7. Kohorn EI. The new FIGO 2000 staging and risk factor scoring system for gestational trophoblastic disease: Description and critical assessment. Int J Gynecol Cancer. 2001;11(1):73-7. doi: 10.1046/j.1525 1438.2001.011001073.x. [PubMed: 11285037].

8. Rustin GJ, Newlands ES, Lutz JM, Holden L, Bagshawe KD, Hiscox JG, et al. Combination but not single-agent methotrexate chemotherapy for gestational trophoblastic tumors increases the incidence of second tumors. J Clin Oncol. 1996;14(10):2769-73. doi: 10.1200/JCO.1996.14.10.2769. [PubMed: 8874338].

9. Cavoretto P, Gentile C, Mangili G, Garavaglia E, Valsecchi L, Spagnolo D, et al. Transvaginal ultrasound predicts delayed response to chemotherapy and drug resistance in stage I low-risk trophoblastic neoplasia. Ultrasound Obstet Gynecol. 2012;40(1):99-105. doi: 10.1002/uog.11097. [PubMed: 22262502].

10. Garavaglia E, Gentile C, Cavoretto P, Spagnolo D, Valsecchi L, Mangili G. Ultrasound imaging after evacuation as an adjunct to beta-hCG monitoring in posthydatidiform molar gestational trophoblastic neoplasia. Am J Obstet Gynecol. 2009;200(4):417 e1-5. doi: 10.1016/j.ajog.2008.11.032. [PubMed: 19200936].

11. Malek M, Moradi B, Mousavi AS, Ahmadinejad N, Kazemi MA, Gity M. Complementary role of ultrasound in management of gestational trophoblastic disease. Iran J Radiol.2015;12(2).e13955. doi:10.5812/iranjradiol.13955. [PubMed: 26060553]. [PubMed Central: PMC4457963].

12. Li L, Wan X, Feng F, Ren T, Yang J, Zhao J, et al. Pulse actinomycin $\mathrm{D}$ as first-line treatment of low-risk post-molar nonchoriocarcinoma gestational trophoblastic neoplasia. BMC Cancer. 2018;18(1):585. doi: 10.1186/s12885-018-4512-5. [PubMed: 29792175]. [PubMed Central: PMC5966914].
13. Burton GJ, Woods AW, Jauniaux E, Kingdom JC. Rheological and physiological consequences of conversion of the maternal spiral arteries for uteroplacental blood flow during human pregnancy. Placenta. 2009;30(6):473-82. doi: 10.1016/j.placenta.2009.02.009. [PubMed: 19375795]. [PubMed Central: PMC2697319].

14. Agarwal R, Strickland S, McNeish IA, Patel DC, Foskett M, Boultbee JE, et al. Doppler ultrasonography of the uterine artery and the response to chemotherapy in patients with gestational trophoblastic tumors. Clin Cancer Res. 2002;8(5):1142-7. [PubMed: 12006530].

15. Agarwal R, Harding V, Short D, Fisher RA, Sebire NJ, Harvey R, et al. Uterine artery pulsatility index: A predictor of methotrexate resistance in gestational trophoblastic neoplasia. Br J Cancer. 2012;106(6):1089-94. doi: 10.1038/bjc.2012.65. [PubMed: 22374461]. [PubMed Central: PMC3304432].

16. Long MG, Boultbee JE, Begent RH, Hanson ME, Bagshawe KD. Preliminary Doppler studies on the uterine artery and myometrium in trophoblastic tumours requiring chemotherapy. Br J Obstet Gynaecol. 1990;97(8):686-9. doi: 10.1111/j.1471-0528.1990.tb16239.x. [PubMed: 2169301].

17. Sita-Lumsden A, Medani H, Fisher R, Harvey R, Short D, Sebire N, et al. Uterine artery pulsatility index improves prediction of methotrexate resistance in women with gestational trophoblastic neoplasia with FIGO score 5-6. BJOG. 2013;120(8):1012-5. doi: 10.1111/1471-0528.12196. [PubMed: 23759086].

18. Oguz S, Sargin A, Aytan H, Kelekci S, Dumanli H. Doppler study of myometrium in invasive gestational trophoblastic disease. Int J Gynecol Cancer. 2004;14(5):972-9. doi: 10.1111/j.1048-891X.2004.14537.x. [PubMed: 15361211]. 